Classification

Physics Abstracts

$71.55 \mathrm{~J}$

\title{
$N$-orbital model for a two-dimensional disordered system in a strong magnetic field
}

\author{
T. S. J. Streit \\ Institut für Theoretische Physik, Ruprecht-Karls-Universität, D-6900 Heidelberg, F.R.G.
}

(Reçu le 2 mai 1984, accepté le 30 mai 1984)

\begin{abstract}
Résumé. - Dans un champ magnétique intense, orthogonal au plan d'un système désordonné bi-dimensionnel, un modèle à $N$ orbitales par site est considéré. L'Hamiltonien est projeté dans le niveau de Landau fondamental. Dans la limite de $N$ grand, le résultat correspond à un col, invariant par translation, d'une théorie des champs. Le développement en puissances de $1 / N$ est effectué diagrammatiquement et la conductivité est calculée jusqu'à l'ordre $1 / N^{2}$. Dans ce développement on n'observe aucun seuil de mobilité. Le résultat est conforme au modèle $\sigma$ non linéaire unitaire; il est indépendant du champ magnétique et de l'intensité du désordre si l'on exprime l'énergie en unités de la largeur de bande.
\end{abstract}

\begin{abstract}
An N-orbital model is considered for a two-dimensional disordered system in a strong perpendicular magnetic field. The Hamiltonian is projected on the lowest Landau level. In a field theoretical formulation the large $N$ limit result corresponds to the translationally invariant saddle point for this model. A diagrammatic expansion in powers of $1 / N$ is performed around the large $N$ limit. The conductivity is calculated up to order $1 / N^{2}$. No mobility edge is found in this expansion. The result is consistent with the non-linear unitary $\sigma$-model and independent of magnetic field and disorder strength if the energy is measured in units of the bandwidth.
\end{abstract}

The effect of a strong magnetic field on the transport properties of a two-dimensional disordered system [1, 2] has been the subject of recent theoretical approaches [2-6] and numerical calculations $[3,7]$. In the absence of magnetic field one knows from localization theory that as dimension two is approached and as the system becomes infinitely large, all states become localized. The critical behaviour of localization is described by the non-linear $\sigma$-model [8-10], even if a weak magnetic field is added [10-11]. The unitary fixed point is stable [12], whereas the orthogonal one becomes unstable if a magnetic field is added.

Nevertheless the experimental results for the quantum Hall effect in a two-dimensional system indicate the existence of a mobility edge. Earlier theoretical work [3] explained the quantum Hall effect by assuming such a mobility edge. Recent theoretical and numerical work [4-7] now shows the existence of extended states at the centre of the band. Levine, Libby and Pruisken [4] obtain for a strong magnetic field the effective Lagrangian of the non-linear $\sigma$-model together with an additional term. They infer that this additional term leads to delocalization in the band centre. It can be written as a total divergence, therefore perturbation theory around the saddle point should be useless for seeing any change due to this extra term. 
Our calculation is restricted to the lowest Landau level, where we define an $N$-orbital model. This restriction is justified for a strong magnetic field, since no mixing between Landau levels is then expected. Introducing disorder through a white noise potential Wegner [13] calculated exactly the one-particle Green's function for the lowest Landau level. Brézin, Gross and Itzykson [14] generalized this result to an arbitrary distribution for the random potential. Combining the techniques of a $1 / N$ expansion from Oppermann, Wegner [15], hereafter denoted as I, with the result for the lowest Landau level from Wegner [13], hereafter denoted as II, we calculate the conductivity up to order $1 / N^{2}$. Hikami [5] recently calculated the diffusion constant for the lowest Landau level at the band centre. He obtained an extended state using a Pade Borel resummation. In a second work [6] he carried out a $1 / N$ expansion. The diagrams for the diffusion constant are expressed in terms of the Euler trails (II) and then numerically evaluated at the band centre. He obtains a localizing logarithmic correction for the diffusion constant. The result is not inconsistent with the unitary non-linear $\sigma$-model. We calculated the correction analytically for the whole band up to order $1 / N^{2}$, also obtaining the logarithmic correction, the energy dependence agreeing with that of the unitary non-linear $\sigma$-model. The conductivity has the form :

$$
\sigma(\omega, E)=\frac{e^{2}}{4 \pi^{2} \hbar} g^{\alpha}(E)\left(1+\frac{1}{\left(g^{\alpha}(E)\right)^{2}} \ln \left(\frac{\omega}{A}\right)\right),
$$

where $g^{\propto}(E)$ is the dimensionless conductance for the large $N$-limit, $\omega$ the external frequency, $E$ the energy, and the meaning of $A$ will become evident later. Hikami obtains a factor 0.34 for the logarithmic correction for the diffusion constant, whereas we obtain 1/4 in our exact calculation.

Our calculation starts out from the translationally invariant saddle point solution $[4,8]$. The expansion up to order $1 / N^{2}$ does not give an indication of the existence of extended states, but yields agreement with the unitary non-linear $\sigma$-model. So it seems that the information about extended states does not appear if only this saddle point is picked out and an expansion is performed around it.

The Hamiltonian for the lowest Landau level is

$$
H=\sum_{\alpha, \beta}^{N} \iint \mathrm{d}^{2} r \mathrm{~d}^{2} r^{\prime}\left(\frac{1}{2} \hbar \omega_{\mathrm{c}} C\left(r, r^{\prime}\right) \delta_{\alpha \beta}+\frac{1}{\sqrt{N}} V_{\alpha \beta}(r) \delta\left(r-r^{\prime}\right)\right)|r \alpha\rangle\left\langle r^{\prime} \beta\right|,
$$

$C$ is the projector on the lowest Landau level. With the vector potential $A=1 / 2 B(-y, x)$ it has the form :

$$
C\left(r, r^{\prime}\right)=\rho_{0} \exp \left\{-\frac{1}{4 l^{2}}\left(\xi \xi^{*}+\xi^{\prime} \xi^{*}-2 \xi^{*} \xi\right)\right\}
$$

where $\xi=x+i y$ and $\rho_{0}=\frac{1}{2 \pi l^{2}}=\frac{e B}{2 \pi \hbar c}$.

To the kets $|r\rangle$ which span Hilbert space, $N$ orbitals labelled by $\alpha$ are added. $v$ is a white noise potential which allows hopping between the orbitals. The ensemble is characterized by the distribution

$$
P(V)=\exp \left\{-\sum_{\alpha, \beta} \frac{1}{2 W} \int \mathrm{d}^{2} r V_{\alpha \delta}(r) V_{\beta \alpha}(r)\right\},
$$

therefore $\overline{V_{\alpha \beta}(r)}=0, \overline{V_{\alpha \beta}(r) V_{\gamma \delta}\left(r^{\prime}\right)}=W \delta_{\alpha \delta} \delta_{\beta \gamma} \delta\left(r-r^{\prime}\right)$.

Contrary to the tight binding model in $\mathrm{I}$, in this continuum model the orbitals lack physical meaning, being introduced to perform an expansion in $1 / N$. Note that in our ensemble $\overline{V_{\alpha \beta}} V_{\beta \alpha} \neq 0$, whereas $\overline{V_{\alpha \beta} V_{\alpha \beta}}=0$ if $\alpha \neq \beta$ (non-diagonal elements are complex), and it is invariant under 
global rotations in the space of orbitals. We choose this ensemble, denoted phase invariant ensemble in $\mathrm{I}$, because time reversal invariance is broken by the magnetic field.

In the large $N$-limit one obtains for the one-particle Green's function

$$
\begin{gathered}
G_{\alpha \beta}^{\alpha}\left(r, r^{\prime}, z\right)=C\left(r, r^{\prime}\right) G^{\alpha}(z) \delta_{\alpha \beta}, \\
G^{\infty}(z)=\frac{1}{\sqrt{W \rho_{0}}}\left(\frac{z-1 / 2 \hbar \omega_{c}}{2 \sqrt{W \rho_{0}}} \pm i s\left(1-\frac{\left(z-1 / 2 \hbar \omega_{c}\right)^{2}}{4 W \rho_{0}}\right)^{1 / 2}\right),
\end{gathered}
$$

where $s=\operatorname{sgn} \operatorname{Im}(z)$. This gives the semicircle law for the density of states. Let us denote

$$
\cos \theta=\frac{z-1 / 2 \hbar \omega_{c}}{2 \sqrt{W \rho_{0}}}, \text { so that } G^{\infty}(z)=\frac{1}{\sqrt{W \rho_{0}}} \mathrm{e}^{-i s \theta}
$$

Then we consider the two-particle Green's function. We will use the following property valid for arbitrary two-particle diagrams; a diagram with external ends $1,2,3,4$ as depicted in figure 1a with $2 n$ factors $V$ exhibits besides factors $\left(z-1 / 2 \hbar \omega_{c}\right)^{-1},\left(z^{\prime}-1 / 2 \hbar \omega_{c}\right)^{-1}, 1 / N$ and $\delta$ factors in the labels $\alpha$ the following dependence on the endpoints

$$
\begin{array}{r}
K\left(\xi_{1}, \xi_{2}, \xi_{3}, \xi_{4}\right)=\frac{\left(W \rho_{0}\right)^{n}}{m_{1}+m_{2}} \exp \left\{-\frac{1}{4 l^{2}} \sum_{i} \xi_{i} \xi_{i}^{*}-2 \frac{m_{1}}{m_{1}+m_{2}}\left(\xi_{3} \xi_{1}+\xi_{4} \xi_{2}\right)-\right. \\
\left.-2 \frac{m_{2}}{m_{1}+m_{2}}\left(\xi_{3}^{*} \xi_{2}+\xi_{4}^{*} \xi_{1}\right)\right\},
\end{array}
$$

where $m_{1}\left(m_{2}\right)$ are the Euler trails for the Feynman graph (II) corresponding to the one-particle diagram obtained by linking end 4 with end 2 (end 4 with end 1 ) in the two-particule diagram of figure la.

In order to obtain the large $N$-limit, the propagators are now replaced by the large $N$-limit ones. Then in this limit only ladder diagrams contribute whose corresponding bubble graphs are shown in figure $1 \mathrm{~b}$. If $m_{1}$ is the number of vertices, then there are $m_{1}$ Euler trails, by linking ends 1 with 2 and there is only one trail by connecting ends 3 with 2 . Using (7) and setting $r_{2}=r_{4}=r^{\prime}$, $r_{1}=r_{3}=r$ we obtain, by Fourier transformation, a geometrical series for the sum over $m_{1}$. This leads to the result

$$
K_{\alpha \beta \beta \alpha}^{\infty}\left(q, z, z^{\prime}\right)=\frac{1}{N} \frac{1}{W} \frac{\mathrm{e}^{-i s \theta-i s \theta^{\prime}} \mathrm{e}^{-1 / 2 l^{2} q^{2}}}{1-\mathrm{e}^{-i s \theta-i s \theta^{\prime}} \mathrm{e}^{-1 / 2 l^{2} q^{2}}} .
$$

This is a surprising result because it resembles that of the local gauge invariant tight-binding model (I, Eq. (5.2)) if we identify $m(q)$ with $\mathrm{e}^{-1 / 2 l^{2} q^{2}}$. For the retarded advanced case we obtain

a)

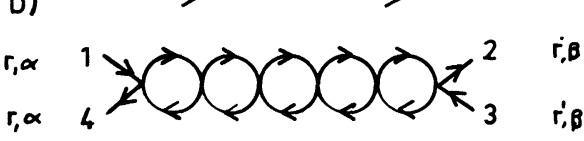

Fig. 1. - Two-particle Green's function, the box stands for an arbitrary diagram. Figure $1 \mathrm{~b}$ is a large $N$ limit graph for the two-particle function. 
in the hydrodynamic limit the diffusive behaviour

$$
K^{\infty}\left(q, z, z^{\prime}\right)=\frac{2 \pi \rho^{\alpha}(E)}{D^{\infty} q^{2}-i s \omega},
$$

where $D^{\infty}=1 / 2 l^{2} W 2 \pi \rho^{\infty}(E)$ and where $\rho^{\infty}(E)$ is the density of states which follows from (6). Using the Einstein relation $\sigma=N e^{2} / \hbar D \rho(E)$, the conductivity follows as

$$
\sigma=N \frac{e^{2}}{2 \pi^{2} \hbar} \sin ^{2} \theta
$$

It is independent of magnetic field and disorder if energy is measured in units of the bandwidth.

The diagrams for higher orders of $1 / N$ are obtained by connecting the $T$ matrices, defined by the sum of all ladder diagrams, with the large $N$-limit Green functions. Then we proceed as in I and denote with $\Lambda$ the sum of all irreducible contributions to the two-particle function. How do we calculate the diagrams for $\Lambda$ ? One way could be replacing the $T$ matrices by one of their ladder contributions, counting the Euler trails, and then summing up all ladder realizations. Hikami did this, numerically evaluating the last step. We use the large $N$-limit result for the sum of the ladders and calculate the momentum dependence of the junctions which connect the $T$ matrices and endpoints. As depicted in figure 2 the junctions in the phase invariant ensemble are roundabout-like. A roundabout with $m$ two-particle functions attached to it has the following dependence on the momenta

$v\left(\mathbf{q}_{1}, \ldots, \mathbf{q}_{m}\right)=4 \pi^{2} \rho_{0} \delta\left(\mathbf{q}_{1}+\mathbf{q}_{2}+\cdots+\mathbf{q}_{m}\right) \times \exp \left\{-\frac{1}{4} l^{2} \sum_{i=1}^{m} q_{i}^{2}+\frac{1}{2} i s l^{2} \sum^{m} \mathbf{q}_{i} \times \mathbf{q}_{j}\right\}$,

where $s=1$ for a clockwise roundabout, and -1 for the opposite case. The momenta are labelled in the clockwise sense. Absorbing the first exponential term into the $T$-matrices we can introduce two-particle propagators as

$$
g_{z z^{\prime}}(q)=\frac{\mathrm{e}^{-i s \theta-i s \theta^{\prime}} \mathrm{e}^{-1 / 2 l^{2} q^{2}}}{1-\mathrm{e}^{-i s \theta-i s \theta^{\prime}} \mathrm{e}^{-1 / 2 l^{2} q^{2}}}
$$

Then besides the phase factors coming from the second term in (11) the functional form for the diagrams is the same as that in $\mathrm{I}$ if we identify $m(q)$ with $\mathrm{e}^{-1 / 2 l^{2} q^{2}}$. Next we would like to apply the partial resummation of the diagrams of $\Lambda$ as was found by Oppermann, Wegner I and which allowed the introduction of reduced vertices. This can be done if the related diagrams in the partial resummation have the same phase factor. This is true as can be proved by comparing the phase factors from two junctions connected by a $T$-matrix and merging the two roundabouts into one. The phase factor is the same in both cases.

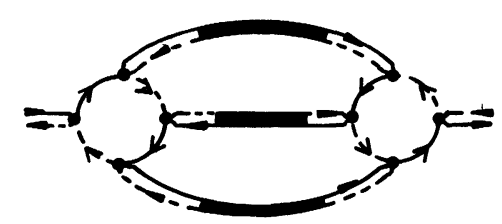

Fig. 2. - Diagram contributing to $\Lambda$ in order $1 / N^{2}$, the boxes stand for the $T$-matrices, which are connected by roundabout junctions. 
There are no diagrams for $\Lambda$ to order $1 / N$. The reduced diagrams to order $1 / N^{2}$, which contribute to the diffusion constant are those denoted with $l_{1}, l_{5}, v_{1}, v_{4}, \mu_{1}, \mu_{2}, v_{1}$ (in I, Fig. 20). The leading order contribution which comes from diagrams $\mu_{2}$ and $v_{4}$ and which goes as $\ln ^{2} \omega$, cancels. In the next leading order $(\ln \omega)$, diagrams $\mu_{2}, v_{4}$ and $t_{5}$ have to be considered. Up to order $q^{2}$ and writing only the term proportional to $\ln \omega$, they give the following contribution

$$
\begin{aligned}
& l_{5}=-\alpha q^{2} l^{2}\left(\frac{\pi^{2}}{6}+2 s\left(\theta-\frac{\pi}{2}\right) \operatorname{tg} s \theta-\frac{1}{2}\left(\zeta^{2}\left(\mathrm{e}^{2 i s \theta}\right)+\zeta^{2}\left(\mathrm{e}^{-2 i s \theta}\right)\right)\right), \\
& \mu_{2}=\alpha q^{2} l^{2}\left(1-\ln (2-2 \cos 2 s \theta)-\frac{1}{2 i} \operatorname{ctg} s \theta\left(\zeta^{2}\left(\mathrm{e}^{2 i s \theta}\right)-\zeta^{2}\left(\mathrm{e}^{-2 i s \theta}\right)\right)\right), \\
& v_{4}=-\alpha q^{2} l^{2}-l_{5}-\mu_{2}
\end{aligned}
$$

with

$$
\alpha=\frac{1}{8}\left(\frac{1}{N \sin ^{2} \theta}\right)^{2} \ln \left(\frac{-i s \omega}{2 \pi W \rho^{\alpha}(E)}\right) \text { and } \zeta^{2}=\sum_{n=1}^{\infty} \frac{z^{n}}{n^{2}} .
$$

The additional phase factors coming from (12) do not lead to any divergent contribution. Then in the next leading order the integrals are formally the same as in reference [7, 9], where an $\varepsilon=d-2$ expansion with $m(q)=1 /\left(1+a q^{2}\right)$ was performed. Besides the different $m(q)$, we have a continuum model, and our calculations are exactly in two dimensions. The external frequency acts as a lower soft cutoff. The individual results for the diagrams differ in both cases. Nevertheless universality shows up in the sum, where every odd energy dependence disappears leaving a term with the dependence of the unitary case. The correction $\delta D / D^{\alpha}$ for the diffusion constant is :

$$
\frac{\delta D}{D^{\propto}}=-\frac{1}{l^{2}} \frac{\partial}{\partial q^{2}}\left(v_{4}+\mu_{2}+l_{5}\right)=2 \alpha .
$$

At the band centre $(\theta=\pi / 2)$, the factor of the logarithm is $1 / 4$ instead of 0.34 obtained by Hikami. Using Einstein's relation we obtain equation (1) for the conductivity with $g(E)=2 N \sin ^{2} \theta$. If energy is measured in units of the bandwidth the conductance is independent of magnetic field and disorder. Up to order $1 / N^{2}$ no mobility edge is found. The localizing logarithmic correction to the large $N$-limit has an energy dependence which is consistent with the unitary nonlinear $\sigma$-model.

\section{Acknowledgments.}

I am indebted to Prof. F. Wegner for suggesting this problem, for helpful discussions and for reading the manuscript. I am grateful to J. Chalker, K. Ziegler and especially to $\mathbf{R}$. Oppermann whom I would like to thank for all the long clarifying discussions on localization and related subjects. This work was supported by the CONICIT (Consejo Nacional de Investigaciones Científicas y Tecnológicas), Venezuela, and mainly by the DAAD (Deutscher Akademischer Austauschdienst), Fed. Rep. of Germany. 


\section{References}

[1] Von Klitzing, K., Tausendfreund, B., Obloh, H. and Herzog, T., p. 1, Aoki, H., p. 11, Hadju, J., p. 23, ENGLERT, T., p. 87, in G. Landwehr (ed.) « Application of high magnetic field in semiconductor Physics » Springer Lecture Notes in Physics 177 (1983).

[2] Halperin, B., Helv. Phys. Acta 56 (1983) 75.

[3] ANDo, T., p. 176, Thouless, D. J., p. 191, in Y. Nagaoka and H. Fukuyama (eds.) « Anderson Localization ", Springer Series in Solid-State-Sciences 39 (1982).

[4] Levine, H., LibBY, S. B. and Pruisken, A. M. M., Phys. Rev. Lett. 51 (1983) 1915 ; and The quantized Hall effect I, II, III, preprints (1984).

[5] Hikami, S., Phys. Rev. B 29 (1984) N. 6.

[6] Hikami, S., Anderson localization of the two dimensional electron in a random potential under a strong magnetic field, preprint (1984).

[7] Schweitzer, L., Kramer, B. and MacKinnon, A., J. Phys. C., to be published.

[8] Schäfer, L., Wegner, F., Z. Phys. B 38 (1980) 113.

[9] Brézin, E., Hikami, S., Zinn-Justin, J., Nucl. Phys. B 165 (1980) 528.

[10] Wegner, F., p. 8, Hikami, S., p. 15, in Y. Nagaoka and H. Fukuyama (eds.) « Anderson Localization ", Springer Series in Solid-State-Sciences 39 (1982).

[11] Jüngling, K., OpPermann, R., Z. Phys. B 38 (1980) 93.

[12] OPPERMANN, R., Ziegler, K., Localization by diffusions preprint (1983).

[13] Wegner, F., Z. Phys. B 51 (1983) 279.

[14] Brézin, E., Gross, D. J., Itzykson, C., Nucl. Phys. B, to be published.

[15] Oppermann, R., Wegner, F., Z. Phys. B 34 (1979) 237. 